\title{
Decision and Control Model for Promoting Public Transit via Lottery Incentives
}

\author{
Tang-Hsien Chang, Yih-Chiun Jiang \\ Department of Civil Engineering, National Taiwan University
}

\begin{abstract}
This article reports on a decision model that highlights a reward-based promotional strategy for a bus organization to maintain its market. The market control law is obtained from an optimal solution in the system equations on the basis of the relationship among the transit operator, ticket agent, and government. The article presents a case study for the Taipei bus transit system. Results in this research confirm the effectiveness of the proposed strategy for bus operators as well as for traffic improvement. The proposed model reveals the optimal actions for the agent and bus operators under governmental policy.
\end{abstract}

\section{Introduction}

Public transit ridership in many urban areas is declining. Passenger cars are preferred for travel, subsequently inducing traffic congestion. Although transportation authorities have implemented several encouraging policies, such as tax deductions and exclusive bus system operations, passengers lack interest in traveling by bus because such transportation policies do not directly benefit the customers. In addition, the elasticity of the price of public transportation is extremely low (Lago, Mayworm, and Mcenroe 1981), with an average range of $-0.28 \pm 0.16$. Such inelastic circumstances imply that reducing the fare price leads to a net loss in revenues. 
Indeed, almost all countries have a regulated pricing scheme for public transportation. Applying various pricing strategies to affect transportation markets directly conflicts with such regulations. Therefore, a stimulatory strategy is expected to ensure the survival of bus operators and increase public transit system use.

This study presents a novel incentive system to exert control on the transportation market. The proposed system focuses on selling prepaid tickets merged with a lottery to satisfy the operator's expectations, particularly in terms of ridership or revenue. A case study is conducted to verify that such a strategy yields a satisfactory solution for bus operators while also alleviating traffic congestion. As a concrete measure in the proposed strategy, the government is to apply a subsidy policy for bus operators when total passenger loads reach a certain threshold within a specific period. Bus operators can also encourage their ticketing agent to promote the use of prepaid tickets by offering a bonus to the agent when the amount of ticket sales achieves a certain quantity. The agent is also offered several reward grades measured in purchased tickets or mileage for passengers. This incentive strategy subsequently stimulates the market.

Control theory is the basic methodology in the analysis of marketing relationships within the proposed promotional system. The upper level of the strategic goal is to alleviate traffic congestion by encouraging individuals to travel by bus. The primary level is to maximize profits for the ticketing agent and the bus operational organization. The proposed model is constructed with the agent's and the bus operators' profits, respectively, in terms of time. The solution identifies the sensitivity and optimality of the control variables.

\section{Premises for Modeling}

\section{Incentive System Structure}

Figure 1 depicts the relationships among the ticketing agent, bus operators, and the government in the incentive system. According to these relationships, the ticket sales agent attempts to obtain maximum profit through a promotional strategy for selling a sufficient quantity of tickets. The agent's profit includes the net revenue from selling tickets and a bonus obtained from the bus operators. The bus operators' profit comes from carrying more passengers and possibly from a government subsidy. To obtain the subsidy, the operator must meet a performance threshold of total passengers carried within a period, $T$. If more 


\section{Figure 1. Relations among Sales Agent, Bus Operators, and Government}

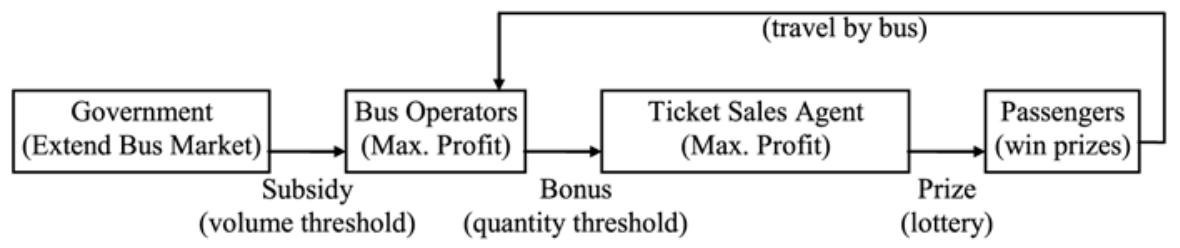

people travel by bus, traffic congestion is reduced and the quality of life in the city improves. Passengers expect to win an incentive prize from the ticketing agent.

\section{Passenger Incentive Types}

Promotional design of the prepaid tickets should be as attractive as possible. To extend the bus transportation market, noncaptive passengers are the targets of the promotional program. According to one survey (Jiang 1998), a lottery is one of the most attractive activities for noncaptive passengers. In this study, a lottery game is designed for the purpose indicated. In the game, both instant and delayed rewards are considered. The quicker an individual purchases a prepaid ticket card the higher the probability of winning an instant reward. After spending the prepaid value of the ticket, the individual has the opportunity to win a grand prize. This strategy encourages individuals not only to purchase prepaid tickets, but also to travel by bus. The structure of the prize layout was arranged as a pyramid with multiple layers and items. The grand prize is generally awarded at the end of a given period, denoted as $T$.

\section{Passenger Demand Function}

During this analysis the passenger demand function is formulated first. Based on the formulated demand function, profits for the ticketing agent and bus operators can then be estimated. Demand is affected by fare and level of transport services such as route, frequency, vehicle-quality (seat, air-conditioned), driver behaviors, and so forth. All routes are assumed to have already been allocated and cannot be changed; the number of existing vehicles is sufficient for any frequency extension (i.e., bus transport capability is far from exhausted and in a depressed period); vehicles are all in the range of usage; and employed drivers are experienced. All people know the fare, route network, schedules, and traffic conditions. The analytical change in price, even through a lottery activity, is relatively small compared to the basic transportation price from the regulated fare. Under these conditions, 
the two factors of ticket price and incentive prize are sufficient to determine the demand variation for most road users. Thus, the demand function is assumed to be linear (McConnell and Brue 1993) in terms of the price and the expectation of winning a prize in the incentives:

$$
q(t)=a p(t)+b E(M)+c
$$

where:
$q(t) \quad$ denotes the volume of bus trips at time $t$
$p(t) \quad$ represents the price of a bus ticket in a unit sale at time $t$, $t \in[0, T]$
$E(M) \quad$ is the expectation of winning a prize $M$
$a, b$ and $c \quad$ are parameters; $a$ must be negative and $b$ be positive

The expectation of winning a prize, of the given incentives, is defined by

$$
E(M)=\sum_{j} M_{j} f\left(M_{j}\right)
$$

where:
$M_{j}$
expresses the prize of jth item of the rewards
$f\left(M_{j}\right)$
is the probability of winning the reward $M_{j}, 0 \leq f\left(M_{j}\right) \leq 1$

\section{Profit Model for a Ticket Sales Agent}

Based on the three premises above, the ticketing agent's profit can be clarified. The ticket agent's profit is calculated as the sum of the net profit from selling tickets and the bonus, with the cost of the prizes issued to lottery winners deducted according to the promotional policy. Assume that the basic price of a ticket is $C$, which the bus consortium (organized by the bus operators in Taipei city) contracts to the agent. The agent sells a unit ticket to a passenger with the price $p(t)$. Thus, the agent's profit at time $t$ is $[p(t)-C] q(t), p(t)>C$. Obtaining $p(t)$ in Equation (1), the profit can be derived as follows:

$$
[p(t)-C] q(t)=\left[\frac{1}{a}(q(t)-b E(M)-c)-C\right] q(t)
$$


The gross gain from ticketing in period $[0, T]$ is

$$
\int_{0}^{T}\left[\frac{1}{a}(q(t)-b E(M)-c)-C\right] q(t)(1+i)^{T-t} d t
$$

where:

$i \quad$ denotes the interest rate

In addition, supposing that the bus consortium sets the threshold for tickets sold at level $H$ for paying a bonus, and the agent gains the bonus $B(\cdot)$ if the tickets are sold out to the amount of $Q(T)$ in period $T$ :

$$
B(Q(T))\left\{\begin{array}{l}
=0, \text { if } Q(T)<H \\
\geq 0, \text { if } Q(T)=H \\
>0, \text { if } Q(T)>H
\end{array}\right.
$$

Equation (5) indicates that the agent's bonus vanishes if ticket sales do not reach the volume $H$. The agent will have a bonus of $B(\cdot)$ if the $H$ volume is sold out. In general, $B(\cdot)$ is designed as a linear function with a marginal bonus while the sold quantity is beyond $H$. However, the expenditure of the agent for the lottery is

$$
E(M)=\sum M_{j} L_{j}=M_{1} L_{1}+M_{2} L_{2}
$$

where:

$$
L_{j} \text { represents the quantity of the reward } j
$$

The first item $(j=1)$ is the expenditure for the instant reward and the second item $(j=2)$ denotes the prize bestowed at the end of the given period of $T$. In considering the instant reward, the right-hand term in Equation (6) could be replaced by

$$
\int_{0}^{T}\left[m_{1}(t) l_{1}(t)(1+i)^{T \cdot t} d t+M_{2} L_{2}\right.
$$

where:

$$
\begin{array}{ll}
m_{1}(t) & \text { denotes the price of an instant reward } \\
l_{1}(t) & \text { represents the quantity of the instant reward }
\end{array}
$$

In brief, Equation (7) is used instead of the following form:

$$
\int_{0}^{T} \hat{M}_{1}(t)(1+i)^{T-t} d t+\hat{M}_{2}
$$


By incorporating Equations (4), (5), and (8), the total gain of the agent yields the following:

$$
\begin{aligned}
G= & \int_{0}^{T}\left\{\left[\frac{1}{a}(q(t)-b E(M)-c)-C\right] q(t)-\hat{M}_{1}(t)\right\}(1+i)^{T-t} d t \\
& +B(Q(T))-\hat{M}_{2}
\end{aligned}
$$

With the maximum profit objective, the extreme value from Equation (9) is obtained:

$$
\begin{aligned}
G^{*}=\max & \int_{0}^{T}\left\{\left[\frac{1}{a}(q(t)-b E(M)-c)-C\right] q(t)-\hat{M}_{1}(t)\right\}(1+i)^{T-t} d t \\
+B(Q(T))-\hat{M}_{2} &
\end{aligned}
$$

While considering the agent's attitude in referring to Equation (5), three possible types of actions are dealt as follows:

1. The agent may discard obtaining the bonus if the threshold of the sold ticket volume is too high to afford. The proposition is written as

$$
\left\{\begin{aligned}
G_{1}^{*}=\max \int_{0}^{T}\left\{\left[\frac{1}{a}(q(t)-b E(M)-c)-C\right] q(t)-\hat{M}_{1}(t)\right\}(1+i)^{T-t} d t \\
-\hat{M}_{2} \\
\text { subject to } \quad q(t) \geq 0, Q(0)=0, Q(T)<H \\
Q(t)=\int_{0}^{t} q(t) d t \text { and } E(M)=\sum_{j} M_{j} f\left(M_{j}\right)
\end{aligned}\right.
$$

2. For Type II, the agent decides that his task is to reach the threshold for getting a bonus. He is not willing to put forth further sales effort due to the low margin for a bonus. The proposition is written as

$$
\left\{\begin{array}{c}
G_{2}^{*}=\max \int_{0}^{T}\left\{\left[\frac{1}{a}(q(t)-b E(M)-c)-C\right] q(t)-\hat{M}_{1}(t)\right\}(1+i)^{T-t} d t \\
+B(Q(T))-\hat{M}_{2} \\
\text { subject to } \quad q(t) \geq 0, Q(0)=0, Q(T)=H \\
Q(t)=\int_{0}^{t} q(t) d t \quad \text { and } \quad E(M)=\sum_{j} M_{j} f\left(M_{j}\right)
\end{array}\right.
$$


3. For Type III, based on the bonus condition in Equation (5) issued by the bus operators, the agent realizes that more profit can be earned by selling more tickets. The proposition is as

$$
\left\{\begin{array}{c}
G_{3}^{*}=\max \int_{0}^{T}\left\{\left[\frac{1}{a}(q(t)-b E(M)-c)-C\right] q(t)-\hat{M}_{1}(t)\right\}(1+i)^{T-t} d t \\
+B(Q(T))-\hat{M}_{2} \\
\text { subject to } \quad q(t) \geq 0, Q(0)=0, Q(T)>H \\
Q(t)=\int_{0}^{t} q(t) d t \text { and } E(M)=\sum_{j} M_{j} f\left(M_{j}\right)
\end{array}\right.
$$

Next, an attempt is made to identify the agent's final decision from the above three propositions. To do so, Equations (11), (12), and (13) must be solved with respect to variable $q(t)$. Since the propositions are dynamic problems depending on time $t$, the optimal control theory is applied (Chiang, 1992; Kamien and Schwartz 1991). Let $\dot{x}=\dot{x}(t)=q(t)$ and

$$
\Omega(x, \dot{x}, t)=\left\{\frac{1}{a}(\dot{x})^{2}-\left(\frac{b}{a} E(M)+\frac{c}{a}+C\right) \dot{x}-\hat{M}_{1}(t)\right\}(1+i)^{T-t}
$$

According to the Euler equation (Kamien and Schwartz 1991), to find the extreme value from Equations (11), (12), and (13), the following function holds:

$$
\frac{\partial \Omega}{\partial x}-\frac{d}{d t} \cdot \frac{\partial \Omega}{\partial \dot{x}}=0
$$

Substituting Equation (14) into the differential Equation (15) yields, at time t,

$$
\dot{x}=q(t)=\frac{1}{2}(b E(M)+c+a C)+C_{0}(1+i)^{t}
$$

This is a general solution form. Different constraints in each type lead to different results. Based on the constraint and boundary conditions in Type I, we obtain the following result:

$$
\dot{x}_{1}(t)=q_{1}(t)=\frac{1}{2}(b E(M)+c+a C)
$$

By integration,

$$
Q_{1}^{*}(T)=\frac{(b E(M)+c+a C) T}{2}
$$


Substitute Equations (17) and (18) into (10) yields

$$
\begin{aligned}
G_{1}^{*}= & \frac{\left(c^{2}+2 a c C+a^{2} C^{2}+2 b E(M) c+2 a b E(M) C+b^{2} E(M)^{2}+4 a \hat{M}_{1}(t)\right)}{4 a \ln (1+i)} \\
\cdot\left[1-(1+i)^{T}\right]-\hat{M}_{2} & \text { (1+i) }
\end{aligned}
$$

as well as from Equation (1),

$$
p_{1}^{*}(t)=\frac{1}{2 a}(a C-b E(M)-c)
$$

Similarly, for Type II, we have

$$
\begin{aligned}
q_{2}^{*}(t) & =\frac{1}{2}(b E(M)+c+a C)+\frac{\left[H-\frac{1}{2}(b E(M)+c+a C) T\right] \ln (1+i)}{\left[(1+i)^{T}-1\right]}(1+i)^{t} \\
Q_{2}^{*}(T) & =H \\
G_{2}^{*}= & \frac{\left(c^{2}+2 a c C+a^{2} C^{2}+2 b E(M) c+2 a b E(M) C+b^{2} E(M)^{2}+4 a \hat{M}_{1}(t)\right)}{4 a \ln (1+i)} \\
& \cdot\left[1-(1+i)^{T}\right]+\frac{(-2 H+a C T+b E(M) T+c T)^{2} \ln (1+i)}{4 a\left[(1+i)^{T}-1\right]}(1+i)^{T} \\
& +B(H)-\hat{M}_{2} \\
p_{2}^{*}(t) & =\frac{1}{2 a}\left\{a C-b E(M)-c+\frac{[2 H-(a C+b E(M)+c) T] \ln (1+i)}{\left[(1+i)^{T}-1\right]}(1+i)^{t}\right\}
\end{aligned}
$$

For Type III, the bonus function, described in previous, could be replaced by

$$
B\left(x\left(t_{f}\right)\right)=B(H)+K\left[x\left(t_{f}\right)-H\right]
$$


and this implies

$$
B^{\prime}\left(x\left(t_{f}\right)\right)=\frac{d B\left(x\left(t_{f}\right)\right)}{d x\left(t_{f}\right)}=K
$$

where:

$x\left(t_{f}\right)$ denotes the total quantity of sales until the termination, $t_{f}=T$

$K \quad$ is the marginal bonus, the incentive for an extra sale

Consequently,

$$
\begin{aligned}
& q_{3}^{*}(t)=\dot{x}_{3}(t)=\frac{1}{2}(b E(M)+c+a C)-\frac{a}{2} K(1+i)^{t-T} \\
& Q_{3}^{*}(T)=\frac{(b E(M)+c+a C) T}{2}-\frac{a K}{2} \frac{\left[1-(1+i)^{-T}\right]}{\ln (1+i)}
\end{aligned}
$$

Then, we obtain the following result:

$$
\begin{aligned}
G_{3}^{*}= & \frac{\left(c^{2}+2 a c C+a^{2} C^{2}+2 b E(M) c+2 a b E(M) C+b^{2} E(M)^{2}+4 a \hat{M}_{1}(t)\right)}{4 a \ln (1+i)} \\
& \cdot\left[1-(1+i)^{T}\right]+\frac{a K^{2}}{4 \ln (1+i)}\left[1-(1+i)^{-T}\right]+B(H)+K\left[Q_{3}(T)-H\right]-\hat{M}_{2}
\end{aligned}
$$

and corresponding to

$$
p_{3}^{*}(t)=\frac{1}{2 a}\left\{a C-b E(M)-c-a K(1+i)^{t-T}\right\}
$$

The optimal action of the agent should be the most profitable one based on the description of the three types under given conditions:

$$
G^{*}=\max \left\{G_{1}^{*}, G_{2}^{*}, G_{3}^{*}\right\}
$$




\section{Profit Model of Bus Operators}

Bus operators are concerned with the profit they will earn so their goal is also maximum profit. The bus operators' profit function is comprised of the net revenue from their service, the amount of the bonus awarded to their agent, and the governmental subsidy due to their contribution to traffic congestion relief. The governmental subsidy is provided only if the total number of busloads during the fiscal period surpasses the regulated threshold. Net revenue in service is the income from the ticketing agent after deduction of the operating costs shared in the processing of the electronic readers on buses.

While assuming that the operating cost shared in processing one ticket is $\hat{f}$ and the net revenue per ticket is $(C-\hat{f})$, during the period [0,T ], the operators' total profit $W$ is calculated by

$$
W=\int_{0}^{T}[C-\hat{f}] q(t)(1+i)^{T-t} d t-B(Q(T))+G P\left(N_{b}\right)
$$

in which, $G P\left(N_{b}\right)$ represents the governmental subsidy:

$$
G P\left(N_{b}\right)\left\{\begin{array}{l}
=0, \text { if } N_{b}<D \\
\beta\left(N_{b}-D\right)>0, \text { if } N_{b} \geq D
\end{array}\right.
$$

where:

$N_{b} \quad$ denotes the total number of loads carried by the bus operators is the unit of subsidy with respect to a load

$D \quad$ is the threshold for the subsidy

Both $\beta$ and $D$ are regulated by the government. Therefore, the object function of the bus companies is set to be maximum profit as follows:

$$
W^{*}=\max \int_{0}^{T}[C-\hat{f}] q(t)(1+i)^{T-t} d t-B(Q(T))+G P\left(N_{b}\right)
$$

Since three types of agent attitudes have been described, the bus operators also have three respected treatments. Substituting Equations (17) and (18), or (21) and (22), or (27) and (28) into Equation (33) subsequently yields

$$
W^{*}=\max \int_{0}^{T}[C-\hat{f}] q_{k}^{*}(t)(1+i)^{T-t} d t-B\left(Q_{k}^{*}(T)\right)+G P\left(N_{b}\right)
$$


where:

$k$ indicates the three types, $k=1,2,3$, respectively

As previous shown in Equation (25), the bonus can be expressed using the following general form:

$$
B(Q(T))=B(H)+K[Q(T)-H]
$$

The bus operators' profit can then be obtained

$$
W^{*}=\int_{0}^{T}[C-f] q_{k}^{*}(t)(1+i)^{T-t} d t-\left\{B(H)+K\left[Q_{k}^{*}(T)-H\right]\right\}+G P\left(N_{b}\right)
$$

If the ticket agent has made his decision, the bus operators' profit can clearly be confirmed.

\section{Calibration of the Demand Function}

A case study is presented for bus operation in Taipei City, where the Taipei Bus Consortium consists of eight bus companies. Passengers pay for bus fare with a prepaid magnetic card. The prepaid ticket cards are sold through a wholesale agent. The agent sells the prepaid ticket cards from ticketing windows distributed throughout Taipei City in convenience stores. To understand the feasibility of implementing the previous model in Taipei City, consumer opinions were investigated using a questionnaire survey. The survey focused largely on understanding demand from and incentives to passengers. The questionnaire was designed to allow travelers to easily state their preferences.

The contents of the questionnaire are: (1) vehicle ownership-motorcycle, passenger car; (2) trip purpose with transportation modal choice and frequencies in the current run; (3) frequency change of riding the bus if the fare varies a $\Delta p$; (4) level of preferences if a lottery is involved in the bus ticketing; and (5) frequency variations of riding the bus corresponding to different prize structures in a ticketing lottery game.

With a 5 percent level of significance in a random sampling of 1,320 Taipei citizens from the phone directory, the statistical results show the following implications:

- The transportation mode distribution for Taipei citizens is one-fifth for buses, and four-fifths for other modes such as motorcycles, passenger vehicles, and 
taxis. This indicates that approximately one-fifth of the citizens are classified as captive bus passengers and four-fifths are noncaptive bus passengers.

- On average, the mean and standard deviation for bus use frequency for captive bus passengers are 13.90 and 5.133 trips per week, respectively. The mean and standard deviation for noncaptive bus passengers are 1.31 and 0.58 trips per week, respectively.

- Approximately 70 percent of all citizens consider purchasing promotional tickets merged with a lottery game. Compared to the current one-fifth of the population that travels by bus, the lottery promotion can enhance public transportation ridership.

- For the original captive passengers, if the ticket fare increases a unit, they will reduce their bus usage by an average of 1.16 trips per week. In the noncaptive group, if the ticket fare decreases a unit, these passengers may expand their bus usage by an average of 0.35 trips per week.

- The likelihood of traveling by bus increases as the expectation of winning a prize increases. The analysis of variance shows that with a 5 percent level of significance with our promotional alternatives, there is nearly no difference between captive and noncaptive passengers in terms of bus use frequency. When the total reward $(\$ 5,000,000)$ is distributed over the structured pyramid proposed for alternative 1 , the mean increase in bus use frequency is 6.39 trips per week and the standard deviation is 7.050 . If the double incentive in alternative 2 is used, the mean increase in bus use frequencies is 7.43 trips per week. If the expectation value increases a unit, the increase in frequency for all samples is 0.013 trips per week.

Based on the survey, the demand function is calibrated as follows:

For captive passengers

$$
\begin{aligned}
\Delta q_{b}= & -1.305 \Delta p_{b}+0.408 \Delta E(M) \\
& (-7.473)^{*} \quad(3.813)^{*} \\
R^{2}= & 0.432^{* *} \quad F=314.415^{* * *}
\end{aligned}
$$

( ${ }^{*}$ the $t$-value, ${ }^{* *}$ the coefficient of determination, ${ }^{* * *}$ the $F$-value) 
For noncaptive bus passengers

$$
\begin{gathered}
\Delta q_{b}=-1.181 \Delta p_{b}+0.495 \Delta E(M) \\
(-6.641) \quad(4.547) \\
R^{2}=0.396 \quad F=307.790
\end{gathered}
$$

For all samples

$$
\begin{gathered}
\Delta q_{b}=-1.240 \Delta p_{b}+0.454 \Delta E(M) \\
(-9.915) \quad(5.936) \\
R^{2}=0.412 \quad F=618.802
\end{gathered}
$$

where:

$\Delta q_{b} \quad$ denotes the quantity variation corresponding to price variation per trip for taking a bus

$\Delta p_{b} \quad$ represents the price variation per trip

$\Delta E(M)$ is the expectation variation for winning a prize

Obviously, from Equations (37) and (38), in light of the price variation, captive passengers are more sensitive than noncaptive passengers. Conversely, in considering expectation variation, noncaptive passengers are more sensitive than captive passengers.

To understand the market tendency under the promotional strategy, the demand function in terms of price and the expectation of winning a prize should be clarified. This demand function can be derived from the difference in Equation (39). Next, Equation (39) is transformed into a step function

$$
q_{b}^{n+1}-q_{b}^{n}=a_{0}\left(p_{b}^{n+1}-p_{b}^{n}\right)+b_{0}\left[E^{n+1}(M)-E^{n}(M)\right]
$$

where:

$$
\begin{aligned}
& a_{0}=-1.24 \\
& b_{0}=0.454
\end{aligned}
$$


In considering the current mean frequencies for taking the bus transit by captive and noncaptive bus passengers, 13.90 and 1.31 trips per week, weighting with onefifth of the total trips for captive and four-fifths for noncaptive, the population mean frequency for taking the bus is estimated to be 3.828 trips per week. In Taipei City, the current bus price is uniformly $\$ 15$ per trip without promotion. Thus, the boundary state values can be set as $q_{b}^{0}=3.828, p_{b}^{0}=15$, and $E^{0}(M)=0$. By mathematical inductive method (Saber 1996), Equation (40) implies

$$
q_{b}(t)=-1.24 p_{b}(t)+0.454 E(M)+22.428
$$

Letting $q_{b}(t)$ be deducted $q_{b}{ }^{0}$, the induced quantity for taking the bus at time $t$, $q_{b}^{+}(t)$, due to the promotion, is calculated as

$$
q_{b}^{+}(t)=-1.24 p_{b}(t)+0.454 E(M)+18.6
$$

Furthermore, if the quantity is expressed by the number of prepaid ticket cards in terms of card price and expectation value, Equation (42) yields

$$
q_{c}^{+}(t)=\frac{1}{N}\left[-1.24 p_{c}(t) / N+0.454 E(M)+18.6\right]
$$

where:

$N \quad$ denotes the number of trips paid using a card

In Taipei City, a ticket card with $\$ 600$ can pay for 40 trips (independent of trip distance). By doing so, the demand function is finally realized as follows:

$$
q_{c}^{+}(t)=-7.75 \times 10^{-4} p_{c}(t)+113.5 \times 10^{-4} E(M)+0.465
$$

Equation (44) represents a person's extra demand trend in unit of card quantity.

\section{Market Analysis}

The market effect is primarily evaluated in terms of the sensitivities and tendencies of the bus consortium and the ticketing agent, as well as efficiency in public transportation. As stated in previous sections, obviously the government controls the period length $T$, subsidy premium $G P\left(N_{b}\right)$, and threshold $D$ for subsidy. The 
bus consortium determines basic ticket price $C$, operating cost $f$, bonus $B(H)$, threshold loads $H$ for receiving bonus, and its margin $K$. Game bucks $M_{1}$ and $M_{2}$ distributed over the structured pyramid layer $L_{1}$ and $L_{2}$ are provided by the ticket agent. These parameters definitely influence the variations in $p^{*}, Q^{*}, G^{*}, W^{*}$, and $N_{b}$. These are clarified as follows:

- Governmental Policy and Domination. Assuming that the government approves and supports the implementation strategy, how much $G P\left(N_{b}\right)$, $D$, and $T$ should be initially announced by the promotional policy? In the following case study, the periodic activity cycle is normally assumed to encompass one year due to the fiscal system. Based on the records for the past two years, the city government sponsored the city bus consortium with $\$ 300$ million annually and with total busloads averaging 650 million trips annually. Therefore, the load subsidy is assumed to be about $\$ 0.5$ per trip. In a moderate case, the government can hopefully increase busloads by 36 million trips a year with the proposed incentive strategy. The threshold $D$ can then be set at 686 million loads ( $=650$ million +36 million) for paying the extra subsidy. This means that if the annual busloads, $N_{b}$, exceed the threshold of 686 million, the bus consortium can obtain an extra $\$ 0.5$ subsidy per load, i.e. $G P^{+}\left(N_{b}^{+}\right)=0.5 N_{b}^{+} . N_{b}^{+}=N_{b}$-650 million. Restated, $G P^{+}\left(N_{b}^{+}\right)$denotes the total extra subsidy based on the extra loads $N_{b}^{+}$over the increased volume $D^{\prime}=36$ million.

- Bus Consortium's Proposition. According to the data from the Taipei City Bus Consortium, their ticketing agent currently receives 4.063 percent of revenue from the selling price. The basic price $C$ issued from the consortium is $\$ 575.62$ per ticket card on account of the selling price of $\$ 600$. Because the government is to pay an extra subsidy at level of threshold $D$, the consortium accordingly decides what threshold $H$ ' of the extra cards sold for the bonus provision proposed to the agent will maximize their own profit under the consideration of slope $K$ (\$/card sold). $H^{\prime}=(\mathrm{H}-650$ million $) / 40$ in that each card can pay for 40 trips. However, $H^{\prime}$ must be equal to or larger than $D^{\prime}$ 140, which is dominated by the government. Based on Equation (36), the bonus is herein designated by $B(Q(T))=K \cdot Q(T)$, if $Q(T)>=H$; otherwise, $B(Q(T))=0 . \hat{f}$ is counted at $\$ 0.76$ per card for processing expenditures.

- Ticketing Agent's Plan. In the case of a promotion for bus passengers, a reward of $\$ 5$ million is provided for the game. Of this total, $\$ 2$ million is for instant rewards uniformly distributed over the whole year, and $\$ 3$ million is for the 
delayed prize, the final lottery reward. Referring to Equation (6) (8), $\hat{M}_{2}(t)$ is estimated about $\$ 38,462$ per week. $\hat{M}_{1}(t)=\$ 3$ million.

- Others. Six percent is taken as a default for the annual interest rate in the following analysis. The expectation $E(M)$ based on $M_{1}$ and $M_{2}$ and proportional to the market volume is calculated iteratively and finalized by the amount of ticket sold $Q(T)$. Refer to Equation (2), $f\left(M_{j}\right)=L_{j} / Q(T)$, for all $j$.

According to official estimates by Taipei, the market has 600,000 attendants. Replacing the personal extra card demand $q_{c}^{+}(t)$ with the market volume in Equation (44) and substituting into $q(t)$ of the model described in section 2 , the optimal card price $p_{c}^{*}$, the total extra quantity of cards sold $Q_{c}^{*+}(T)$, total extra gain of the agent $G^{*+}$, and total extra net revenue of the bus consortium $W^{*+}$ will then be calculated for each type under previous parameters, consciousness, and assumptions. Figure 2(a) illustrates the agent's maximal extra gain curves $G^{*+}$ with respect to the threshold $H^{\prime}$ under the condition of marginal bonus $K=\$ 6$ per card sold. Figure 2(b), (c), and (d) display the relevant plots related to the optimal card price, total extra profit for the bus consortium, and total extra quantity of passengers loaded $\left(N_{b}^{+}=40 \cdot Q_{c}^{*+}(T)\right)$, respectively. According to Figure 2(a), the agent's reaction is obviously based on what $H^{\prime}$ ' was provided by the consortium when calculating the maximal gain from one of three actions: Types I, II, or III.

When the threshold of extra cards sold for receiving the bonus from the bus consortium is less than 1.07 million (i.e., $H^{\prime}<1.07$ million), the agent's best action is type III, selling more ticket cards earns him more money. If the threshold is provided between 1.07 million and 1.46 million, the best action is type II, in which the agent's policy is to sell the ticket cards just to hit the threshold. Otherwise, $H^{\prime}>1.46$ million, the best action is type I, not having interest in the bonus provided. Therefore, the decision curve must be the bold envelope line-the linked line that each segment meets along the shapes or tangents to the spheres-indicated in Figure 2(a). Under this circumstance, the corresponding curve in Figure 2(c) for the bus consortium's profit is also bold.

From the bus consortium's perspective, however, the maximal profit point occurs at $H^{\prime}=1.46$ million and the agent's action should be type II. Because $H^{\prime}=1.46 \mathrm{mil}-$ lion is the turning point of an agent's decision for type I or II, the bus consortium should lower the threshold slightly from 1.46 million to ensure the agent's locking at type II. The final equilibrium point between the bus consortium and the agent leads to the optimal ticket card price of $\$ 598$ (Figure 2(b)), with total extra loads being 58.4 million (Figure 2(d)). The extra profit for the bus consortium would be 
Figure 2. Solution of the Presented Case

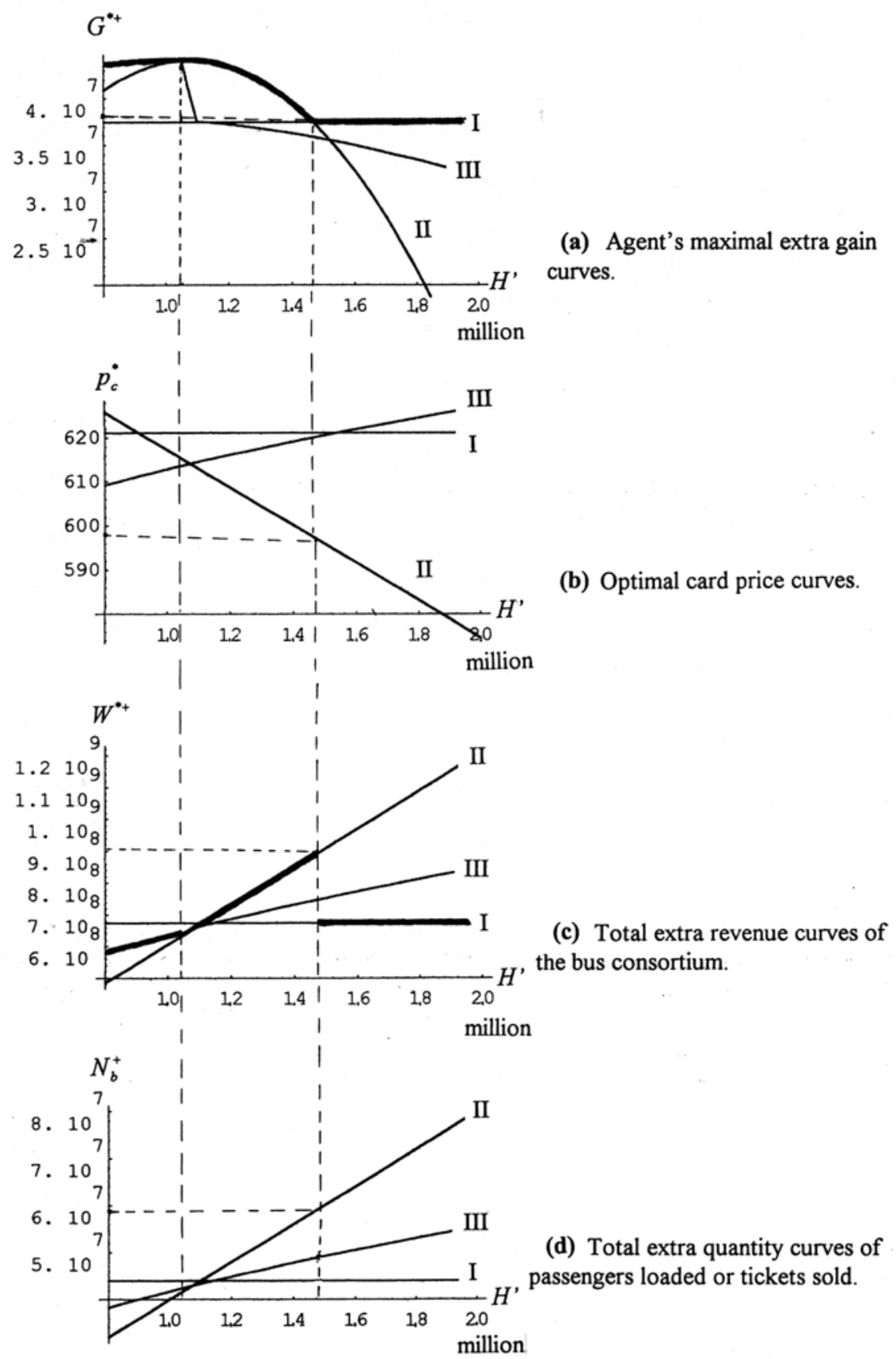


$\$ 885,299,000$ (Figure 2(c)), which includes the subsidy of $\$ 29.2$ million from the government, supposing that the threshold of extra loads $D$ ' for subsidy receipt is announced at 36 million by the government. The maximal extra gain by the agent at this equilibrium point is $\$ 37,707,100$ (Figure $2(a)$ ).

Table 1 summarizes the equilibrium results of the cases: $D^{\prime}=36$ million, 54 million and 72 million with respect to $K=0,2,4,6,8$, and 10 . The table reveals that if the government sets the load threshold at 36 million for bus consortium subsidization, the agent can easily achieve the target only via lottery strategy $\left(N_{b}^{+}>36\right.$ million), despite any bus consortium bonus incentive, even $K=0$ (no incentive).

\section{Table 1. Equilibrium Results of the Taipei Case in Presented Model}

\begin{tabular}{|c|c|c|c|c|c|c|}
\hline $\begin{array}{c}D^{\prime} \\
\text { (million } \\
\text { trips) }\end{array}$ & $\begin{array}{c}K \\
(\$)\end{array}$ & $\begin{array}{c}H^{\prime} \\
\text { (million } \\
\text { cards) }\end{array}$ & $\begin{array}{c}W^{*+} \\
(\$)\end{array}$ & $\begin{array}{c}G^{*+} \\
(\$)\end{array}$ & $\begin{array}{c}p_{c}^{*} \\
(\$)\end{array}$ & $\begin{array}{c}N_{b}^{+} \\
\text {(million } \\
\text { tickets) }\end{array}$ \\
\hline \multirow{5}{*}{36.0} & 0 & 1.09 & $667,528,000$ & $37,516,000$ & 613 & 43.6 \\
\cline { 2 - 8 } & 2 & 1.26 & $769,090,000$ & $37,547,600$ & 606 & 50.4 \\
\cline { 2 - 8 } & 4 & 1.37 & $833,477,000$ & $37,585,200$ & 601 & 54.8 \\
\cline { 2 - 8 } & 6 & 1.46 & $885,299,000$ & $37,707,100$ & 598 & 58.4 \\
\cline { 2 - 8 } & 8 & 1.55 & $936,762,000$ & $37,498,800$ & 594 & 62.0 \\
\cline { 2 - 8 } & $\mathbf{1 0}$ & $\mathbf{1 . 6 2}$ & $\mathbf{9 7 5 , 8 1 9 , 0 0 0}$ & $\mathbf{3 7 , 8 2 8 , 4 0 0}$ & $\mathbf{5 9 1}$ & $\mathbf{6 4 . 8}$ \\
\hline \hline \multirow{5}{*}{54.0} & 0 & 1.09 & $645,728,000$ & $37,516,000$ & 613 & 43.6 \\
\cline { 2 - 8 } & 2 & 1.26 & $743,890,000$ & $37,547,600$ & 606 & 50.4 \\
\cline { 2 - 8 } & 4 & 1.37 & $833,477,000$ & $37,585,200$ & 601 & 54.8 \\
\cline { 2 - 8 } & 6 & 1.46 & $885,299,000$ & $37,707,100$ & 598 & 58.4 \\
\cline { 2 - 7 } & 8 & 1.55 & $936,762,000$ & $37,498,800$ & 594 & 62.0 \\
\cline { 2 - 7 } & $\mathbf{1 0}$ & $\mathbf{1 . 6 2}$ & $\mathbf{9 7 5 , 8 1 9 , 0 0 0}$ & $\mathbf{3 7 , 8 2 8 , 4 0 0}$ & $\mathbf{5 9 1}$ & $\mathbf{6 4 . 8}$ \\
\hline \hline \multirow{5}{*}{72.0} & 0 & 1.09 & $645,728,000$ & $37,516,000$ & 613 & 43.6 \\
\cline { 2 - 7 } & 2 & 1.26 & $743,890,000$ & $37,547,600$ & 606 & 50.4 \\
\cline { 2 - 7 } & 4 & 1.37 & $806,077,000$ & $37,585,200$ & 601 & 54.8 \\
\cline { 2 - 7 } & 6 & 1.46 & $856,099,000$ & $37,707,100$ & 598 & 58.4 \\
\cline { 2 - 7 } & 8 & 1.55 & $905,762,000$ & $37,498,800$ & 594 & 62.0 \\
\cline { 2 - 7 } & 10 & 1.62 & $943,419,000$ & $37,828,400$ & 591 & 64.8 \\
\hline
\end{tabular}


Obviously, the agent will raise the ticket price to earn more profit if $K$ is below $\$ 4$. If the government sets the load threshold at 54 million for bus consortium subsidization, the agent meets the target merely on the condition that $K$ is greater than $\$ 4$. In addition, if the government sets the load threshold at 72 million, the agent cannot achieve the target if $K<=\$ 10$. However, the agent hopes that $K$ is as large as possible while the bus consortium prefers a lower $D^{\prime}$. According to Table 1 , from the perspectives of the agent and bus consortium, $D^{\prime}=54$ million through 36 million is acceptable. Meanwhile, $K=\$ 10$ is the best solution for their profits, with $\$ 37,828,400$ extra gain for the agent and $\$ 975,819,000$ for the consortium. Finally, bus trips would be increased by about 64.8 million annually.

\section{Conclusions}

This article presented a novel promotion strategy for public transit, with particular emphasis on strategy efficiency through an incentive system between passengers and ticketing agents, ticket agents and bus operators, and bus operators and the government. The mathematical model is formulated with a methodology of optimal control description of the market for this incentive system. This model focuses largely on maximizing the public transit market. Both the ticket agent and bus companies receive their maximal profits in an equilibrium market. Results obtained from this strategy can successfully enhance public transportation.

According to this study, a lottery game for bus passengers is very attractive to the people in Taipei City. Although the captive bus passengers are sensitive to changes in ticket price, both captive and noncaptive passengers reflect the same concerns about rewarding expectations. With the analysis in a previous model, clearly the proposed incentive strategy reveals the efficiency of traffic improvement. While public transport patronage is gradually decreasing, this study's considerations are a useful contribution.

\section{References}

Chiang, A.C. 1992. Elements of dynamic optimization. New York: McGraw-Hill, Inc.

Jiang, Y.-C. 1998. A rewarding system study for saving the bus transportation market. Master Thesis (in Chinese), Tamkang University, Taipei, Taiwan. 
Kamien, M. I., and N. L. Schwartz. 1991. Dynamic optimization, 2nd ed. Elsevier Science Publishing Co.

Lago, A. M., P. D. Mayworm, and J. M. Mcenroe. 1981. Transit service elasticity: evidence from demonstrations and demand models. Journal of Transport Economics and Policy 14: 99-119.

McConnell, C. R., and R. L. Brue. 1993. Economics-principles, problems, and policies. New York: McGraw-Hill, Inc.

Saber, N. E. 1996. An introduction to difference equations. New York: Springer-Verlag, Inc.

\section{About the Authors}

TANG-Hsien Chang (thchang@ntu.edu.tw) is a professor of civil engineering at National Taiwan University. He received a B.S. degree in statistics from National Cheng-Chi University, Taipei, and M.S. degree in civil engineering and Ph.D. degree from National Taiwan University, Taipei.

YIH-ChIUN JIANG (cyc626@ms41.hinet.net) is an operational engineer at Taipei MRT company. He received an M.S. degree in transportation management from Tamkang University, Taiwan. 